\title{
Sexual Recombination in the Botrytis cinerea Populations in Hungarian Vineyards
}

\author{
Kálmán Z. Váczy, Erzsébet Sándor, Levente Karaffa, Erzsébet Fekete, Éva Fekete, Mariann Árnyasi, \\ Levente Czeglédi, György J. Kövics, Irina S. Druzhinina, and Christian P. Kubicek
}

First author: Research Institute for Viticulture and Enology, H-3301 Eger, Kölyuktetö, P.O. Box 83, Hungary; second, fifth, and eighth authors: Department of Plant Protection, Centre for Agricultural Sciences, Faculty of Agriculture, University of Debrecen, H-4032 Böszörményi út 138, Debrecen, Hungary; third and fourth authors: Department of Genetics and Applied Microbiology, Faculty of Science and Technology, University of Debrecen, H-4010, P.O. Box 56, Debrecen, Hungary; sixth and seventh authors: Institute of Animal Sciences, Centre for Agricultural Sciences, Faculty of Agriculture, University of Debrecen, H-4032 Böszörményi út 138; and ninth and tenth authors: Vienna University of Technology, Institute of Chemical Engineering, Division of Gene Technology and Applied Biochemistry, Getreidemarkt 9/E1665, A-1060 Vienna, Austria. First and second authors contributed equally to this manuscript. Accepted for publication 11 August 2008.

\section{ABSTRACT}

Váczy, K. Z., Sándor, E., Karaffa, L., Fekete, E., Fekete, É., Árnyasi, M., Czeglédi, L., Kövics, G. J., Druzhinina, I. S., and Kubicek, C. P. 2008. Sexual recombination in the Botrytis cinerea populations in Hungarian vineyards. Phytopathology 98:1312-1319.

Botrytis cinerea (anamorph of Botryotinia fuckeliana) causes gray mold on a high number of crop plants including grapes. In this study, we investigated the genetic properties of a grape pathogenic population of $B$. cinerea in the area of Eger, Hungary. A total of 109 isolates from 12 areas were sampled. Based on the sequence of the $\beta$-tubulin (tub1) locus, they all belong to group II, a phylogenetic species within $B$. cinerea. Seventyfour isolates were classified as transposa, with both the Flipper and Boty transposons, and 10 were classified as vacuma, lacking both transposons. The remaining isolates contained either only Flipper (13) or Boty (12). Multilocus analysis of sequences from $t u b 1$ and two other loci (elonga- tion factor 1-alpha, tefl, and a minisatellite from the intron of an ATPase, $M S B 1)$ led to poor phylogenetic resolution of strains in individual clades. Analysis of five microsatellites $(\mathrm{Bc} 2, \mathrm{Bc} 3, \mathrm{Bc} 5, \mathrm{Bc} 6$, and $\mathrm{Bc} 10)$ resulted in 55 microsatellite haplotypes within the 109 strains. No correlation was detected among individual haplotypes and the presence/absence of Flipper and/or Boty, the geographic origin, or the year of isolation. Application of the index of association, the chi-square test, and the phi test consistently indicated that the population of Hungarian isolates of $B$. cinerea undergoes sexual reproduction. However, the index of association test suggested the presence of some clonality, and the fixation index showed a low or occasionally moderate level of fixation in the Flipper populations. We conclude that the $B$. cinerea populations in Hungary consist of a strongly recombining group II phylogenetic species.

Additional keywords: transposable elements.
Botrytis cinerea Pers.:Fr., the anamorph of Botryotinia fuckeliana (de Bary ex de Bary) Whetzel, causes gray mold on a number of crop plants in the temperate zones worldwide including grapes (17). In grape vineyards, the frequent occurrence of $B$. cinerea prior to harvest results in serious losses of fruits and deterioration of wine quality. $B$. cinerea was for a long time believed to have no host specificity (17) and to consist of a single anamorphic population whose somatic and phenotypic diversity was due to aneuploidy (6) and heterokaryosis (2,21). In contrast, the application of multiple gene genealogies (8) showed that $B$. cinerea in fact consists of two phylogenetic species (named group I and group II). In group I, no recombination was detected (though clonality was not demonstrated either), it showed a reduced host range, and lacked the two transposable elements that were characterized from B. cinerea Boty (5) and Flipper (20). Group II, in contrast, exhibited strong recombination, showed a wider host range, and most strains contained both transposons. The presence (transposa genotype) or absence (vacuma genotype) of these two transposons in isolates of group II has been associated with differences in the growth rate and conidiospore size $(8,23)$ but does not seem to influence the virulence or host specificity.

Corresponding author: L. Karaffa: E-mail address: 1karaffa@delfin.unideb.hu

doi:10.1094/PHYTO-98-12-1312

(c) 2008 The American Phytopathological Society
B. cinerea is already resistant against most fungicides, thus making alternative strategies for its control essential. A clue to these strategies lies in the understanding of the genetic structure and dynamics of its populations. Interestingly, only a few such studies have been published $(8,11,24,27)$.

The area around the town of Eger, Hungary, is a major and traditional wine growing region in Central Europe where $B$. cinerea is considered to be the third most important grapevine pathogen after downy mildew (Plasmopara viticola/Berk. \& Curt ex de Bary/Berl. \& de Toni) and powdery mildew (Erysiphe necator Schwein. var. necator [syn. Uncinula necator/Schwein./ Burrill var. necator]), with an estimated annual loss of up to 15 to $20 \%$.

The objectives of the present study were to determine the structure of $B$. cinerea populations, which are occurring as vineyard pathogens in this area, and to compare these findings with those in French and Chilean vineyards.

\section{MATERIALS AND METHODS}

Isolation. Strains of $B$. cinerea were collected from vineyards in various locations of the Eger wine districts (Fig. 1). They were isolated from infected berries in 2003 and 2004 during the vintage period (September to October). Single-spore isolates were prepared from each strain and maintained on potato dextrose agar (PDA). Conidial suspensions were stored in 50\% glycerol at $-80^{\circ} \mathrm{C}$. Numbering of strains was done according to the 
chronology of collection, irrespective to the local provenance (Table 1).

DNA isolation. DNA was extracted from aerial mycelium of $B$. cinerea strains grown on PDA medium for 10 days at $20^{\circ} \mathrm{C}$. Magnalyser (Roche, Osterode, Germany) was used for the disruption of fungal cells, and DNA was isolated with the Plant DNA Purification Kit (Qiagen, Hilden, Germany).

Detection of transposable elements Boty and Flipper. Genomic DNA (200 ng) was spotted onto positive charged nylon membrane (Roche), denaturated in $0.5 \mathrm{M} \mathrm{NaOH}$ and $1.5 \mathrm{M} \mathrm{NaCl}$ for $20 \mathrm{~min}$, and neutralized in $0.5 \mathrm{M}$ Tris- $\mathrm{HCl}(\mathrm{pH} 7.5)$ and $1.5 \mathrm{M}$ $\mathrm{NaCl}$ for $20 \mathrm{~min}$. The membrane was equilibrated in $20 \times \mathrm{SSC}(1 \times$ $\mathrm{SSC}$ is $0.15 \mathrm{M} \mathrm{NaCl}$ plus $0.015 \mathrm{M}$ sodium citrate) for $5 \mathrm{~min}$, baked at $120^{\circ} \mathrm{C}$ for $30 \mathrm{~min}$, and prehybridized in DIG Easy Hyb (Roche) for $30 \mathrm{~min}$. Hybridizations were performed overnight at $42^{\circ} \mathrm{C}$ in the same solution after addition of Boty (648 bp) or Flipper (1,250 bp) probe. Primers used were LTR98 (5'-AGCCTGTAGAATCACCAACG-3') and LTR728 (5'-CGGTATTTCTGGTTGGCA-3') for Boty and F300 (5'-GCACAAAACCTACAGAAGA-3') and F1500 (5'-ATTCGTTTCTTGGACTGTA-3') for Flipper $(20,27)$. The amplification protocol consisted of an initial denaturation step $\left(3 \mathrm{~min}, 95^{\circ} \mathrm{C}\right)$ followed by five cycles with a denaturation $\left(1 \mathrm{~min}, 95^{\circ} \mathrm{C}\right)$, an annealing $\left(1 \mathrm{~min}, 60^{\circ} \mathrm{C}\right)$, and an elongation step $\left(1 \mathrm{~min} 72^{\circ} \mathrm{C}\right)$, and 30 slightly different subsequent cycles with a denaturation $\left(1 \mathrm{~min}, 90^{\circ} \mathrm{C}\right)$, an annealing $\left(1 \mathrm{~min}, 60^{\circ} \mathrm{C}\right)$, and an elongation step $\left(1 \mathrm{~min}, 72^{\circ} \mathrm{C}\right)$. Reaction was completed with a final 15 min elongation step at $72^{\circ} \mathrm{C}$. Probes were DIG-labeled with the polymerase chain reaction (PCR) DIG Probe Synthesis Kit (Roche), following the manufacturer's protocol.

PCR amplification and sequencing of single copy genes. A portion of tefl was amplified and sequenced with primers EF1 (5'-ATGGGTAAGGA(A/G)GACAAGAC-3') and EF2 (5'GGA(G/A)GTACCAGT(G/C)ATCATGTT-3') (16); a portion of tubl was amplified and sequenced with primers 155 (5'-CAACCTTCAAAATGCGTGAG-3') and 1174 (5'-AGATGGGTTGCTGAGCTTCA-3') (8); and a fragment of the intron of the ATPase gene atpl containing the minisatellite MSB1 with primers MSB1fw (5'-AAGTTGCTGGTTCCTTGA-3') and MSB1rev (5'GTTGCAACCGGCGTAGAT-3') (10). Purified PCR products were subjected to automatic sequencing at MWG-Biotech AG, Ebersberg, Germany. Sequences were edited manually and deposited in NCBI GenBank (accession nos. EU194917 to EU194951).

Sequence analysis. DNA sequences were aligned first with Clustal X 1.81 (33) and then visually adjusted with Genedoc 2.6 (29). Molecular phylogenies were analyzed with MEGA 3.1 (19), PhyML (13; provided online by PHYML), and Mr. Bayes version 3.0B4 (14). For the latter, MODELTEST3-06 (MODELTEST is available online) was used to compare the likelihood of different nested models of DNA substitution and select the best-fit

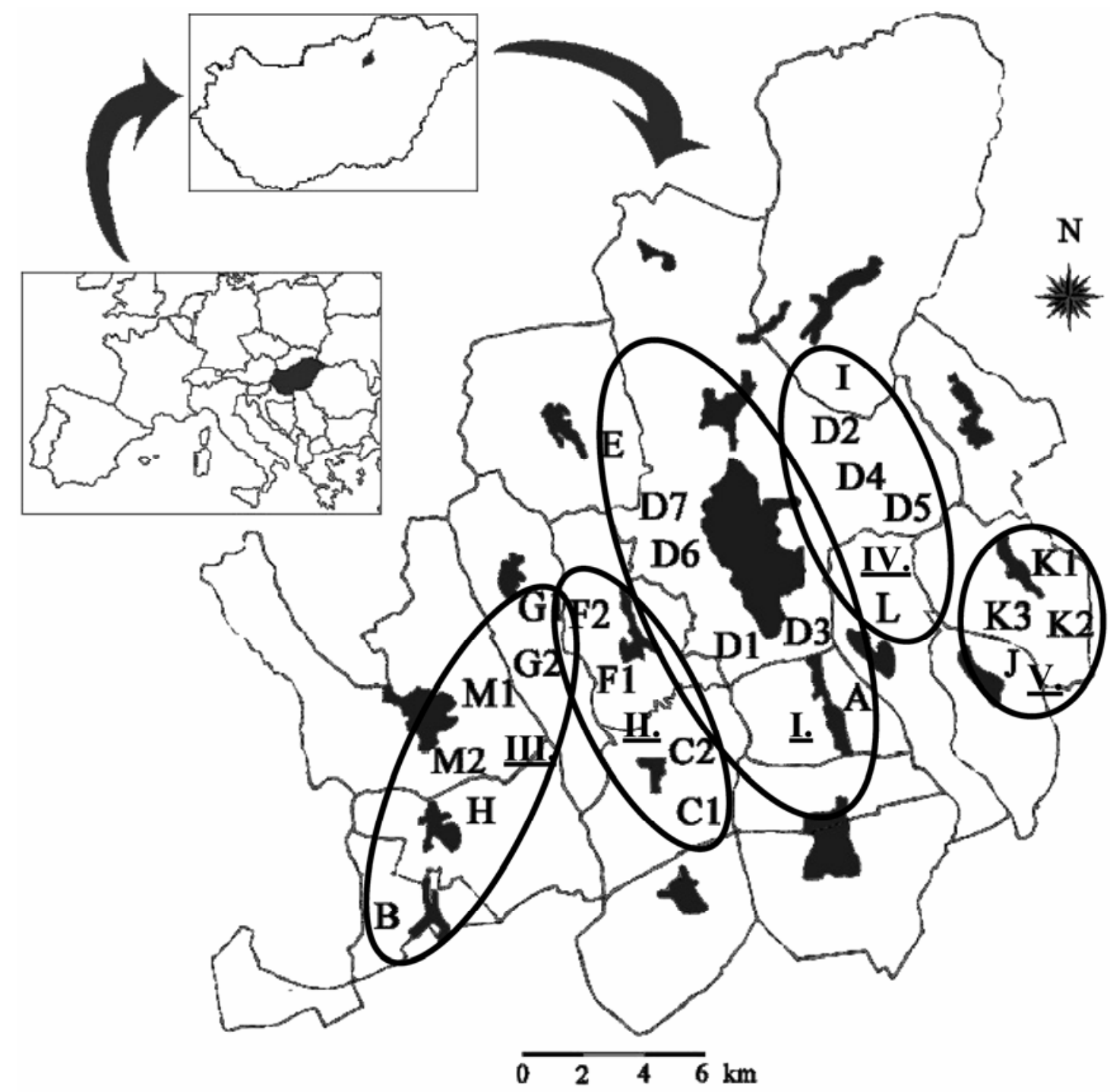

Fig. 1. Geographic map of the Eger sampling regions. Capital letters specify the subregions, as given in Table 1. Circles with italic numbers (I to V) indicate the larger, more homogenous regions (valleys) used for the fixation index $\left(\mathrm{F}_{\mathrm{ST}}\right)$ determinations (Table 3$)$. 
model for the investigated data set using Akaike information criterion (AIC) output strategies. For construction of haplotype networks, sequences were collapsed to haplotypes with DNASp (32). Sequences, one per each haplotype, were aligned and converted to a NEXUS file, and the networks computed by statistical parsimony analysis as implemented in TCS version 2.11 (4).

Microsatellite amplification and analysis. Five microsatellites $(\mathrm{Bc} 2, \mathrm{Bc} 3, \mathrm{Bc} 6, \mathrm{Bc} 7$, and $\mathrm{Bc} 10)$ were analyzed. Amplification protocols and the primers used were identical to those described by Fournier et al. (9). The fragment analysis was performed in an automated single-capillary genetic analyzer (ABI PRISM 310 Genetic Analyzer). The 5' ends of the forward primers were labeled fluorescently (Bc2: FAM, Bc3: NED, Bc6: NED, Bc7:FAM, Bc10: VIC) as suggested by the analyzer's manufacturer.

Detection of recombination and population differentiation. Recombination within $B$. cinerea isolates was tested by (i) the index of association $\left(\mathrm{I}_{\mathrm{A}}\right)$ test, which measures whether the alleles from the different loci in a population are randomly or nonrandomly associated in the analyzed genomes (18), and which was computed with the linkage disequilibrium analysis available on the MLST website (published online by Multi Locus Sequence Typing), and significance was gauged from 1,000 random permutations of the data; (ii) the maximum chi-square test (25), with the modification that all expected $>2$ are considered (30); and (iii) the phi test as implemented in SplitsTree version 3.8 (15). This approach is particularly useful in distinguishing recurrent mutation from recombination when assumptions such as a single, randomly mating, and constant-size population are not met.

Computation of the fixation index $\left(\mathrm{F}_{\mathrm{ST}}\right)$ as a measure of population differentiation was done with POPGENE32 version 1.31 (provided online by the University of Alberta, CA) and by calculating $\mathrm{G}_{\mathrm{ST}}$, which is equal to the weighted average of $\mathrm{F}_{\mathrm{ST}}$ for all multiple alleles (28).

TABLE 1. Botrytis cinerea strains, origin of location, haplotypes, and accession numbers

\begin{tabular}{|c|c|c|c|c|c|c|c|c|c|c|c|c|c|c|c|c|c|}
\hline \multirow[b]{2}{*}{ Year } & \multirow[b]{2}{*}{ Grape } & \multirow[b]{2}{*}{$\begin{array}{c}\text { Strain } \\
\text { no. }\end{array}$} & \multicolumn{2}{|c|}{ Haplotype } & \multirow[b]{2}{*}{$\begin{array}{c}\mathrm{Bc} 2 \\
\mathrm{msat}^{\mathrm{a}}\end{array}$} & \multirow[b]{2}{*}{$\begin{array}{l}\mathrm{Bc} 3 \\
\text { msat }\end{array}$} & \multirow[b]{2}{*}{$\begin{array}{l}\text { Bc6 } \\
\text { msat }\end{array}$} & \multirow[b]{2}{*}{$\begin{array}{l}\text { Bc7 } \\
\text { msat }\end{array}$} & \multirow[b]{2}{*}{$\begin{array}{l}\text { Bc10 } \\
\text { msat }\end{array}$} & \multirow{2}{*}{$\begin{array}{c}\text { Presence } \\
\text { of } \\
\text { Flipper }^{b}\end{array}$} & \multirow[b]{2}{*}{$\begin{array}{c}\text { Presence } \\
\text { of Boty }\end{array}$} & \multicolumn{2}{|c|}{$M S B 1$} & \multicolumn{2}{|c|}{ tefl1 } & & $u b 1$ \\
\hline & & & $\begin{array}{l}\text { Multi- } \\
\text { locus }\end{array}$ & $\begin{array}{l}\text { Trans- } \\
\text { poson }\end{array}$ & & & & & & & & $\begin{array}{l}\text { Haplo- } \\
\text { type }\end{array}$ & $\begin{array}{l}\text { Accession } \\
\text { number }\end{array}$ & $\begin{array}{l}\text { Haplo- } \\
\text { type }\end{array}$ & $\begin{array}{c}\text { Accession } \\
\text { number }\end{array}$ & $\begin{array}{l}\text { Haplo- } \\
\text { type }\end{array}$ & $\begin{array}{c}\text { Accession } \\
\text { number }\end{array}$ \\
\hline 2003 & Zenit & 3 & 1 & 1 & 166 & 217 & 116 & 118 & 179 & 1 & 1 & 1 & EU194924 & 4 & EU194925 & 2 & EU194936 \\
\hline 2003 & Olaszrizling & 9 & 2 & 2 & 166 & 223 & 116 & 118 & 165 & 1 & 1 & 2 & EU194920 & 4 & EU194925 & 4 & EU194938 \\
\hline 2003 & Leányka & 13 & 2 & 3 & 166 & 219 & 116 & 110 & 179 & 1 & 0 & 2 & EU194921 & 4 & EU194925 & 4 & EU194938 \\
\hline 2003 & Olaszrizling & 17 & 2 & 4 & 160 & 221 & 116 & 114 & 181 & 1 & 1 & 2 & EU194921 & 4 & EU194925 & 4 & EU194941 \\
\hline 2003 & Hárslevelü & 18 & 26 & 5 & 168 & 211 & 160 & 110 & 171 & 0 & 1 & 3 & EU194923 & 4 & EU194926 & 9 & EU194948 \\
\hline 2003 & Medina & 22 & 2 & 6 & 160 & 219 & 116 & 118 & 181 & 1 & 1 & 2 & EU194921 & 4 & EU194928 & 4 & EU194941 \\
\hline 2003 & Zenit & 29 & 10 & 7 & 168 & 219 & 120 & 116 & 187 & 1 & 1 & 4 & EU194917 & 2 & EU194927 & 4 & EU194938 \\
\hline 2003 & Zenit & 30 & 15 & 8 & 166 & 213 & 116 & 110 & 179 & 1 & 1 & 2 & EU194921 & 3 & EU194929 & 4 & EU194938 \\
\hline 2003 & Olaszrizling & 31 & 7 & 9 & 166 & 221 & 116 & 118 & 165 & 1 & 0 & 4 & EU194917 & 4 & EU194925 & 5 & EU194943 \\
\hline 2003 & Olaszrizling & 32 & 15 & 8 & 166 & 213 & 116 & 110 & 179 & 1 & 1 & 2 & EU194921 & 3 & EU194929 & 4 & EU194938 \\
\hline 2003 & Leányka & 33 & 3 & 10 & 166 & 221 & 116 & 118 & 181 & 1 & 1 & 2 & EU194921 & 4 & EU194925 & 10 & EU194949 \\
\hline 2003 & Leányka & 34 & 8 & 9 & 166 & 221 & 116 & 118 & 165 & 1 & 1 & 4 & EU194917 & 4 & EU194925 & 4 & EU194941 \\
\hline 2003 & Kékfarnkos & 36 & 9 & 11 & 166 & 221 & 116 & 116 & 165 & 1 & 1 & 4 & EU194917 & 4 & EU194925 & 12 & EU194951 \\
\hline 2003 & Olaszrizling & 38 & 4 & 12 & 166 & 219 & 116 & 110 & 181 & 1 & 1 & 2 & EU194921 & 4 & EU194925 & 1 & EU194932 \\
\hline 2003 & Hárslevelü & 41 & 6 & 13 & 168 & 219 & 116 & 116 & 181 & 1 & 0 & 2 & EU194921 & 4 & EU194925 & 3 & EU194936 \\
\hline 2003 & Eger csillaga & 43 & 10 & 14 & 166 & 219 & 116 & 118 & 181 & 1 & 1 & 4 & EU194917 & 2 & EU194927 & 4 & EU194938 \\
\hline 2003 & Leányka & 45 & 1 & 15 & 166 & 213 & 116 & 118 & 179 & 1 & 1 & 1 & EU194922 & 4 & EU194925 & 2 & EU194937 \\
\hline 2003 & Leányka & 46 & 2 & 9 & 166 & 221 & 116 & 118 & 165 & 1 & 1 & 2 & EU194921 & 4 & EU194925 & 4 & EU194942 \\
\hline 2003 & Leányka & 47 & 24 & 16 & 160 & 219 & 116 & 116 & 177 & 0 & 1 & 1 & EU194922 & 2 & EU194927 & 4 & EU194938 \\
\hline 2003 & Leányka & 49 & 15 & 17 & 166 & 217 & 116 & 110 & 179 & 1 & 1 & 2 & EU194921 & 3 & EU194929 & 4 & EU194933 \\
\hline 2003 & Kékoportó & 53 & 8 & 18 & 160 & 221 & 116 & 118 & 181 & 1 & 0 & 4 & EU194917 & 4 & EU194925 & 4 & EU194938 \\
\hline 2003 & Kékoportó & 54 & 2 & 19 & 168 & 217 & 116 & 116 & 183 & 1 & 1 & 2 & EU194921 & 4 & EU194925 & 4 & EU194938 \\
\hline 2003 & Kékoportó & 55 & 17 & 20 & 180 & 219 & 116 & 110 & 177 & 1 & 1 & 2 & EU194921 & 2 & EU194927 & 4 & EU194938 \\
\hline 2003 & Leányka & 56 & 16 & 21 & 166 & 217 & 116 & 118 & 169 & 1 & 1 & 2 & EU194921 & 3 & EU194929 & 2 & EU194936 \\
\hline 2003 & Leányka & 57 & 26 & 22 & 168 & 211 & 128 & 110 & 173 & 0 & 1 & 3 & EU194923 & 4 & EU194926 & 9 & EU194948 \\
\hline 2003 & Leányka & 58 & 8 & 23 & 166 & 219 & 116 & 118 & 165 & 1 & 1 & 4 & EU194917 & 4 & EU194925 & 4 & EU194938 \\
\hline 2003 & Leányka & 59 & 27 & 24 & 168 & 211 & 116 & 118 & 173 & 0 & 0 & 3 & EU194923 & 4 & EU194926 & 4 & EU194938 \\
\hline 2003 & Leányka & 60 & 8 & 25 & 168 & 219 & 116 & 116 & 177 & 1 & 1 & 4 & EU194917 & 4 & EU194925 & 4 & EU194938 \\
\hline 2003 & Leányka & 61 & 12 & 25 & 168 & 219 & 116 & 116 & 177 & 1 & 1 & 4 & EU194918 & 4 & EU194925 & 6 & EU194945 \\
\hline 2003 & Kékfrankos & 63 & 11 & 26 & 166 & 219 & 116 & 114 & 179 & 1 & 1 & 4 & EU194917 & 3 & EU194929 & 2 & EU194936 \\
\hline 2003 & Leányka & 64 & 15 & 27 & 180 & 217 & 116 & 118 & 181 & 1 & 1 & 2 & EU194921 & 3 & EU194929 & 4 & EU194938 \\
\hline 2003 & Leányka & 65 & 2 & 14 & 166 & 219 & 116 & 118 & 181 & 1 & 0 & 2 & EU194921 & 4 & EU194925 & 4 & EU194938 \\
\hline 2003 & Leányka & 67 & 2 & 11 & 166 & 221 & 116 & 116 & 165 & 1 & 1 & 2 & EU194921 & 4 & EU194925 & 4 & EU194938 \\
\hline 2003 & Zengö & 68 & 3 & 11 & 166 & 221 & 116 & 116 & 165 & 0 & 1 & 2 & EU194921 & 4 & EU194925 & 10 & EU194949 \\
\hline 2003 & Zengö & 70 & 10 & 28 & 164 & 219 & 116 & 110 & 165 & 1 & 1 & 4 & EU194918 & 2 & EU194927 & 4 & EU194938 \\
\hline 2003 & Hárslevelü & 75 & 25 & 29 & 180 & 219 & 116 & 118 & 189 & 1 & 1 & 1 & EU194922 & 3 & EU194928 & 4 & EU194938 \\
\hline 2003 & Hárslevelü & 76 & 8 & 11 & 166 & 221 & 116 & 116 & 165 & 1 & 1 & 4 & EU194918 & 4 & EU194925 & 4 & EU194938 \\
\hline 2003 & Kékfrankos & 86 & 8 & 30 & 180 & 219 & 116 & 116 & 181 & 1 & 1 & 4 & EU194917 & 4 & EU194925 & 4 & EU194938 \\
\hline 2004 & Leányka & 401 & 19 & 31 & 166 & 219 & 116 & 116 & 183 & 1 & 0 & 1 & EU194922 & 4 & EU194925 & 4 & EU194940 \\
\hline 2004 & Leányka & 402 & 19 & 31 & 166 & 219 & 116 & 116 & 183 & 0 & 0 & 1 & EU194922 & 4 & EU194925 & 4 & EU194938 \\
\hline 2004 & Chardonnay & 405 & 1 & 1 & 166 & 217 & 116 & 118 & 179 & 0 & 1 & 1 & EU194924 & 4 & EU194925 & 2 & EU194936 \\
\hline 2004 & Blauburger & 407 & 18 & 32 & 180 & 219 & 122 & 110 & 177 & 0 & 0 & 1 & EU194922 & 5 & EU194930 & 4 & EU194939 \\
\hline 2004 & Blauburger & 408 & 18 & 32 & 180 & 219 & 122 & 110 & 177 & 0 & 1 & 1 & EU194922 & 5 & EU194930 & 4 & EU194938 \\
\hline 2004 & Néró & 409 & 8 & 33 & 166 & 221 & 116 & 116 & 181 & 1 & 1 & 4 & EU194918 & 4 & EU194925 & 4 & EU194938 \\
\hline 2004 & Néró & 410 & 8 & 33 & 166 & 221 & 116 & 116 & 181 & 1 & 0 & 4 & EU194918 & 4 & EU194925 & 4 & EU194938 \\
\hline 2004 & Néró & 411 & 8 & 33 & 166 & 221 & 116 & 116 & 181 & 0 & 1 & 4 & EU194918 & 4 & EU194925 & 4 & EU194938 \\
\hline 2004 & Pinot noir & 412 & 14 & 34 & 170 & 217 & 150 & 110 & 171 & 0 & 0 & 3 & EU194919 & 1 & EU194928 & 9 & EU194948 \\
\hline 2004 & Pinot noir & 413 & 14 & 35 & 170 & 219 & 150 & 110 & 171 & 0 & 0 & 3 & EU194919 & 1 & EU194928 & 9 & EU194948 \\
\hline 2004 & Chardonnay & 414 & 24 & 36 & 180 & 219 & 116 & 118 & 177 & 0 & 0 & 1 & EU194922 & 2 & EU194927 & 4 & EU194938 \\
\hline 2004 & Chardonnay & 415 & 10 & 37 & 180 & 219 & 116 & 114 & 177 & 0 & 0 & 4 & EU194918 & 2 & EU194927 & 4 & EU194938 \\
\hline 2004 & $\begin{array}{l}\text { Sauvignon } \\
\text { blanc }\end{array}$ & 417 & 10 & 38 & 160 & 219 & 120 & 120 & 181 & 0 & 0 & 4 & EU194918 & 2 & EU194927 & 4 & EU194938 \\
\hline 2004 & $\begin{array}{l}\text { Cabernet } \\
\text { sauvignon }\end{array}$ & 419 & 8 & 54 & 166 & 219 & 116 & 116 & 181 & 1 & 1 & 4 & EU194918 & 4 & EU194925 & 4 & EU194938 \\
\hline
\end{tabular}

${ }^{a} \mathrm{Bc} 2, \mathrm{Bc} 3, \mathrm{Bc} 6, \mathrm{Bc} 7$, and $\mathrm{Bc} 10$ msat indicate the five microsatellites analyzed in this study.

b Presence or absence of a particular transposon is indicated by 1 or 0 , respectively. 


\section{RESULTS}

The Hungarian $B$. cinerea populations consist entirely of group II. Fournier et al. (8) recently applied the genealogical concordance phylogenetic species recognition concept (GCPSR) to divide $B$. cinerea into two phylogenetic species, groups I and II. Since the two groups were clearly distinguishable in all four genes used, we used one of the genes from their investigation (tubl) to assign the strains from our study to these two groups. Up to 109 strains from 12 locations (Fig. 1) were thus sampled and sequenced. TBLASTN analysis against the NCBI database indicated that they all were identical to group II strains sequenced by Fournier et al. (8).

Transposon genotypes in the Hungarian B. cinerea group II species. $B$. cinerea group II has been described to mostly exhibit the transposa genotype (i.e., contain both Boty and Flipper) and occasionally the vacuma genotype (i.e., contain neither). To determine whether this also applies to the Hungarian population, we tested for the presence of both transposons in 109 strains by the dot blot hybridization method. The results documented that indeed the vast majority of isolates (74, corresponding to $67.8 \%$ ) contained both Flipper and Boty and was thus transposa. However, some of isolates also contained only Flipper (11.9\%) (13) or only Boty (11\%) (12), respectively. Ten isolates (9.1\%) were vacuma, i.e., they contained neither transposon.

Multilocus structure of the Hungarian B. cinerea group II population. In order to determine the population structure of the Hungarian $B$. cinerea isolates, we sequenced two more loci: a fragment of the elongation factor $1-\alpha$ encoding gene tefl, containing the fourth and fifth intron; and the minisatellite-containing intron of the atpl gene encoding an ATPase. A BLAST search against the $B$. cinerea genome database confirmed that

\begin{tabular}{|c|c|c|c|c|c|c|c|c|c|c|c|c|c|c|c|c|c|}
\hline \multirow[b]{2}{*}{ Year } & \multirow[b]{2}{*}{ Grape } & \multirow[b]{2}{*}{$\begin{array}{c}\text { Strain } \\
\text { no. }\end{array}$} & \multicolumn{2}{|c|}{ Haplotype } & \multirow[b]{2}{*}{$\begin{array}{c}\mathrm{Bc} 2 \\
\mathrm{msat}^{\mathrm{a}}\end{array}$} & \multirow[b]{2}{*}{$\begin{array}{l}\mathrm{Bc} 3 \\
\text { msat }\end{array}$} & \multirow[b]{2}{*}{$\begin{array}{c}\text { Bc6 } \\
\text { msat }\end{array}$} & \multirow[b]{2}{*}{$\begin{array}{l}\mathrm{Bc} 7 \\
\text { msat }\end{array}$} & \multirow[b]{2}{*}{$\begin{array}{l}\text { Bc10 } \\
\text { msat }\end{array}$} & \multirow{2}{*}{$\begin{array}{l}\text { Presence } \\
\text { of } \\
\text { Flipper }^{\mathrm{b}}\end{array}$} & \multirow[b]{2}{*}{$\begin{array}{c}\text { Presence } \\
\text { of Boty }\end{array}$} & & MSBI & & tefl1 & & $u b 1$ \\
\hline & & & $\begin{array}{l}\text { Multi- } \\
\text { locus }\end{array}$ & $\begin{array}{l}\text { Trans- } \\
\text { poson }\end{array}$ & & & & & & & & $\begin{array}{l}\text { Haplo- } \\
\text { type }\end{array}$ & $\begin{array}{l}\text { Accession } \\
\text { number }\end{array}$ & $\begin{array}{l}\text { Haplo- } \\
\text { type }\end{array}$ & $\begin{array}{l}\text { Accession } \\
\text { number }\end{array}$ & $\begin{array}{l}\text { Haplo- } \\
\text { type }\end{array}$ & $\begin{array}{c}\text { Accession } \\
\text { number }\end{array}$ \\
\hline 2004 & $\begin{array}{l}\text { Sauvignon } \\
\text { blanc }\end{array}$ & 421 & 19 & 39 & 168 & 219 & 116 & 110 & 179 & 1 & 1 & 1 & EU194922 & 4 & EU194926 & 4 & EU194938 \\
\hline 2004 & Turán & 423 & 19 & 40 & 166 & 219 & 116 & 116 & 179 & 1 & 1 & 1 & EU194922 & 4 & EU194925 & 4 & EU194938 \\
\hline 2004 & Turán & 424 & 19 & 40 & 166 & 219 & 116 & 116 & 179 & 1 & 1 & 1 & EU194922 & 4 & EU194925 & 4 & EU194938 \\
\hline 2004 & Rizlingszilváni & 425 & 1 & 1 & 166 & 217 & 116 & 118 & 179 & 1 & 1 & 1 & EU194922 & 4 & EU194925 & 2 & EU194936 \\
\hline 2004 & Cabernet franc & 427 & 8 & 31 & 166 & 219 & 116 & 116 & 183 & 1 & 1 & 4 & EU194918 & 4 & EU194925 & 4 & EU194938 \\
\hline 2004 & Kékfrankos & 431 & 13 & 1 & 166 & 217 & 116 & 118 & 179 & 1 & 1 & 4 & EU194918 & 4 & EU194926 & 2 & EU194936 \\
\hline 2004 & Kékfrankos & 432 & 20 & 41 & 180 & 219 & 116 & 116 & 179 & 1 & 1 & 1 & EU194922 & 4 & EU194926 & 7 & EU194946 \\
\hline 2004 & Kékfrankos & 433 & 19 & 41 & 180 & 219 & 116 & 116 & 179 & 1 & 1 & 1 & EU194922 & 4 & EU194926 & 4 & EU194938 \\
\hline 2004 & Kékfrankos & 435 & 10 & 42 & 166 & 221 & 122 & 118 & 181 & 0 & 0 & 4 & EU194918 & 2 & EU194927 & 4 & EU194938 \\
\hline 2004 & Kékfrankos & 437 & 8 & 43 & 166 & 219 & 116 & 120 & 181 & 1 & 1 & 4 & EU194918 & 4 & EU194926 & 4 & EU194938 \\
\hline 2004 & Kékfrankos & 438 & 13 & 44 & 166 & 217 & 116 & 120 & 179 & 1 & 1 & 4 & EU194918 & 4 & EU194926 & 2 & EU194936 \\
\hline 2004 & Kékfrankos & 439 & 8 & 12 & 166 & 219 & 116 & 110 & 181 & 1 & 1 & 4 & EU194918 & 4 & EU194926 & 4 & EU194938 \\
\hline 2004 & Merlot & 440 & 25 & 45 & 160 & 221 & 116 & 118 & 175 & 0 & 1 & 1 & EU194922 & 3 & EU194928 & 4 & EU194938 \\
\hline 2004 & Merlot & 441 & 25 & 45 & 160 & 221 & 116 & 118 & 175 & 0 & 1 & 1 & EU194922 & 3 & EU194928 & 4 & EU194938 \\
\hline 2004 & Kékfrankos & 442 & 1 & 17 & 166 & 217 & 116 & 110 & 179 & 1 & 1 & 1 & EU194922 & 4 & EU194926 & 2 & EU194936 \\
\hline 2004 & Kékfrankos & 443 & 8 & 54 & 166 & 219 & 116 & 116 & 181 & 1 & 1 & 4 & EU194918 & 4 & EU194926 & 4 & EU194938 \\
\hline 2004 & Chaslas & 445 & 8 & 30 & 180 & 219 & 116 & 116 & 181 & 0 & 0 & 4 & EU194918 & 4 & EU194925 & 4 & EU194938 \\
\hline 2004 & Olaszrizling & 448 & 19 & 46 & 166 & 217 & 116 & 116 & 181 & 1 & 1 & 1 & EU194922 & 4 & EU194926 & 4 & EU194944 \\
\hline 2004 & Rizlinszilváni & 449 & 1 & 1 & 166 & 217 & 116 & 118 & 179 & 1 & 1 & 1 & EU194922 & 4 & EU194926 & 2 & EU194936 \\
\hline 2004 & Rizlinszilváni & 450 & 1 & 1 & 166 & 217 & 116 & 118 & 179 & 1 & 1 & 1 & EU194922 & 4 & EU194926 & 2 & EU194936 \\
\hline 2004 & Rizlinszilváni & 451 & 1 & 1 & 166 & 217 & 116 & 118 & 179 & 1 & 1 & 1 & EU194922 & 4 & EU194926 & 2 & EU194936 \\
\hline 2004 & Hárslevelü & 454 & 1 & 47 & 166 & 217 & 116 & 110 & 169 & 1 & 1 & 1 & EU194922 & 4 & EU194926 & 2 & EU194936 \\
\hline 2004 & Hárslevelü & 455 & 19 & 48 & 166 & 219 & 116 & 110 & 183 & 1 & 1 & 1 & EU194922 & 4 & EU194926 & 4 & EU194938 \\
\hline 2004 & Hárslevelü & 456 & 19 & 49 & 166 & 219 & 116 & 118 & 183 & 1 & 1 & 1 & EU194922 & 4 & EU194926 & 4 & EU194938 \\
\hline 2004 & Hárslevelü & 457 & 23 & 50 & 166 & 219 & 116 & 120 & 183 & 0 & 1 & 1 & EU194922 & 4 & EU194926 & 11 & EU194950 \\
\hline 2004 & Hárslevelü & 458 & 22 & 58 & 166 & 219 & 116 & 110 & 183 & 0 & 1 & 1 & EU194922 & 4 & EU194926 & 8 & EU194947 \\
\hline 2004 & Bianka & 460 & 1 & 15 & 166 & 213 & 116 & 118 & 179 & 1 & 1 & 1 & EU194922 & 4 & EU194925 & 2 & EU194936 \\
\hline 2004 & Chardonnay & 461 & 5 & 15 & 166 & 213 & 116 & 118 & 179 & 1 & 1 & 2 & EU194921 & 4 & EU194925 & 2 & EU194936 \\
\hline 2004 & Merlot & 464 & 8 & 14 & 166 & 219 & 116 & 118 & 181 & 1 & 1 & 4 & EU194918 & 4 & EU194925 & 4 & EU194938 \\
\hline 2004 & Chaslas & 465 & 2 & 11 & 166 & 221 & 116 & 116 & 165 & 1 & 1 & 2 & EU194921 & 4 & EU194925 & 4 & EU194938 \\
\hline 2004 & Chaslas & 466 & 2 & 51 & 166 & 219 & 116 & 116 & 165 & 1 & 1 & 2 & EU194921 & 4 & EU194925 & 4 & EU194938 \\
\hline 2004 & Kékfrankos & 468 & 8 & 33 & 166 & 221 & 116 & 116 & 181 & 1 & 1 & 4 & EU194918 & 4 & EU194925 & 4 & EU194938 \\
\hline 2004 & $\begin{array}{l}\text { Cabernet } \\
\text { sauvignon }\end{array}$ & 471 & 2 & 52 & 164 & 219 & 116 & 118 & 181 & 1 & 0 & 2 & EU194921 & 4 & EU194926 & 4 & EU194938 \\
\hline 2004 & Hárslevelü & 473 & 19 & 14 & 166 & 219 & 116 & 118 & 181 & 1 & 0 & 1 & EU194922 & 4 & EU194925 & 4 & EU194938 \\
\hline 2004 & Hárslevelü & 474 & 19 & 14 & 166 & 219 & 116 & 118 & 181 & 1 & 0 & 1 & EU194922 & 4 & EU194925 & 4 & EU194938 \\
\hline 2004 & Hárslevelü & 475 & 2 & 23 & 166 & 219 & 116 & 118 & 165 & 1 & 1 & 2 & EU194921 & 4 & EU194925 & 4 & EU194938 \\
\hline 2004 & Hárslevelü & 476 & 2 & 31 & 166 & 219 & 116 & 116 & 183 & 1 & 1 & 2 & EU194921 & 4 & EU194926 & 4 & EU194935 \\
\hline 2004 & Hárslevelü & 478 & 8 & 11 & 166 & 221 & 116 & 116 & 165 & 1 & 1 & 4 & EU194918 & 4 & EU194925 & 4 & EU194938 \\
\hline 2004 & Kékfrankos & 488 & 21 & 53 & 164 & 213 & 116 & 118 & 181 & 0 & 1 & 1 & EU194922 & 4 & EU194926 & 5 & EU194934 \\
\hline 2004 & Kékfrankos & 489 & 10 & 54 & 166 & 219 & 116 & 116 & 181 & 1 & 1 & 4 & EU194918 & 2 & EU194927 & 4 & EU194938 \\
\hline 2004 & Kékfrankos & 490 & 10 & 14 & 166 & 219 & 116 & 118 & 181 & 1 & 1 & 4 & EU194918 & 2 & EU194927 & 4 & EU194938 \\
\hline 2004 & Kékfrankos & 491 & 19 & 11 & 166 & 221 & 116 & 116 & 165 & 1 & 1 & 1 & EU194922 & 4 & EU194925 & 4 & EU194938 \\
\hline 2004 & Pinot blanc & 4107 & 8 & 11 & 166 & 221 & 116 & 116 & 165 & 1 & 0 & 4 & EU194918 & 4 & EU194925 & 4 & EU194938 \\
\hline 2004 & Olaszrizling & 4147 & 2 & 54 & 166 & 219 & 116 & 116 & 181 & 1 & 1 & 2 & EU194921 & 4 & EU194925 & 4 & EU194938 \\
\hline 2004 & Turán & 4149 & 5 & 15 & 166 & 213 & 116 & 118 & 179 & 1 & 0 & 2 & EU194921 & 4 & EU194925 & 2 & EU194936 \\
\hline 2004 & Turán & 4150 & 2 & 33 & 166 & 221 & 116 & 116 & 181 & 1 & 0 & 2 & EU194921 & 4 & EU194925 & 4 & EU194938 \\
\hline 2004 & Turán & 4151 & 2 & 33 & 166 & 221 & 116 & 116 & 181 & 1 & 1 & 2 & EU194921 & 4 & EU194925 & 4 & EU194938 \\
\hline 2004 & Turán & 4152 & 2 & 33 & 166 & 221 & 116 & 116 & 181 & 1 & 1 & 2 & EU194921 & 4 & EU194925 & 4 & EU194938 \\
\hline 2004 & Leányka & 4163 & 2 & 14 & 166 & 219 & 116 & 118 & 181 & 1 & 1 & 2 & EU194921 & 4 & EU194925 & 4 & EU194938 \\
\hline 2004 & Leányka & 4165 & 2 & 9 & 166 & 221 & 116 & 118 & 165 & 1 & 1 & 2 & EU194921 & 4 & EU194925 & 4 & EU194938 \\
\hline 2004 & Leányka & 4167 & 2 & 55 & 166 & 223 & 116 & 114 & 165 & 1 & 1 & 2 & EU194921 & 4 & EU194925 & 4 & EU194938 \\
\hline 2004 & Leányka & 4168 & 2 & 51 & 166 & 219 & 116 & 116 & 165 & 1 & 1 & 2 & EU194921 & 4 & EU194925 & 4 & EU194938 \\
\hline 2004 & Leányka & 4171 & 2 & 31 & 166 & 219 & 116 & 116 & 183 & 1 & 1 & 2 & EU194921 & 4 & EU194931 & 4 & EU194938 \\
\hline 2004 & Leányka & 4183 & 2 & 9 & 166 & 221 & 116 & 118 & 165 & 1 & 1 & 2 & EU194921 & 4 & EU194931 & 4 & EU194938 \\
\hline 2004 & Olaszrizling & 4185 & 2 & 49 & 166 & 219 & 116 & 118 & 183 & 1 & 1 & 2 & EU194921 & 4 & EU194931 & 4 & EU194938 \\
\hline 2004 & Olaszrizling & 4186 & 2 & 49 & 166 & 219 & 116 & 118 & 183 & 1 & 1 & 2 & EU194921 & 4 & EU194931 & 4 & EU194938 \\
\hline 2004 & Rizlingszilváni & 4188 & 19 & 40 & 166 & 219 & 116 & 116 & 179 & 1 & 1 & 1 & EU194922 & 4 & EU194926 & 4 & EU194938 \\
\hline
\end{tabular}


these two and the tubl gene were unlinked and thus suitable for the analysis. Both Tajima's D-test as well as Fu's F-statistic did not reject the hypothesis of neutral evolution (Table 2), and we therefore considered the gene data set as appropriate for phylogenetic analysis.

The three loci included a total of 1,007 sites of which 26 were polymorphic (Table 2). However, only a low number of sites were parsimony informative. Maximum likelihood trees for individual loci are shown in Figure 2. Four, five, and twelve clades were obtained from the analysis of $M S B 1$, tefl, and tubl, respectively. Likelihoods (loglk) were determined as $-836.45626,-578.61042$, and -578.61042 for $t e f 1$, tubl, and $M S B 1$, respectively. Most branches in these trees were only poorly supported, however, and also did not contain the same isolates, indicating incongruence between different trees. A consistent result was obtained when the individual gene sequences were collapsed to haplotypes and these analyzed by statistical parsimony (4). The corresponding networks are given in Figure 3, again indicating little hierarchical structure within them.

In order to learn whether any of these haplotypes would correlate with the presence or absence of Boty or Flipper, we mapped their absence/presence on the haplotype networks. The data,

TABLE 2. Nucleotide characteristics of Botrytis cinerea tef1, tub1, and MSB1

\begin{tabular}{|c|c|c|c|c|}
\hline Characteristics & tefl & $M S B 1$ & tubl & Total \\
\hline No of sites without gaps & 249 & 254 & 504 & 1,007 \\
\hline No. of polymorphic sites & 6 & 6 & 14 & 26 \\
\hline Parsimony informative sites & 7 & 6 & 5 & 18 \\
\hline $\mathrm{Pi}$ & 0.00384 & 0.00875 & 0.00120 & \\
\hline Theta & 0.00530 & 0.00445 & 0.00513 & \\
\hline Haplotypes number & 5 & 4 & 12 & \\
\hline Haplotype diversity & $0.364[ \pm 0.055]$ & $0.697[ \pm 0.013]$ & $0.448[ \pm 0.052]$ & \\
\hline Fu and Li's D* & 1.182 & 1.108 & -3.783 & \\
\hline Tajima's D & -0.630 & 2.129 & -2.035 & \\
\hline
\end{tabular}
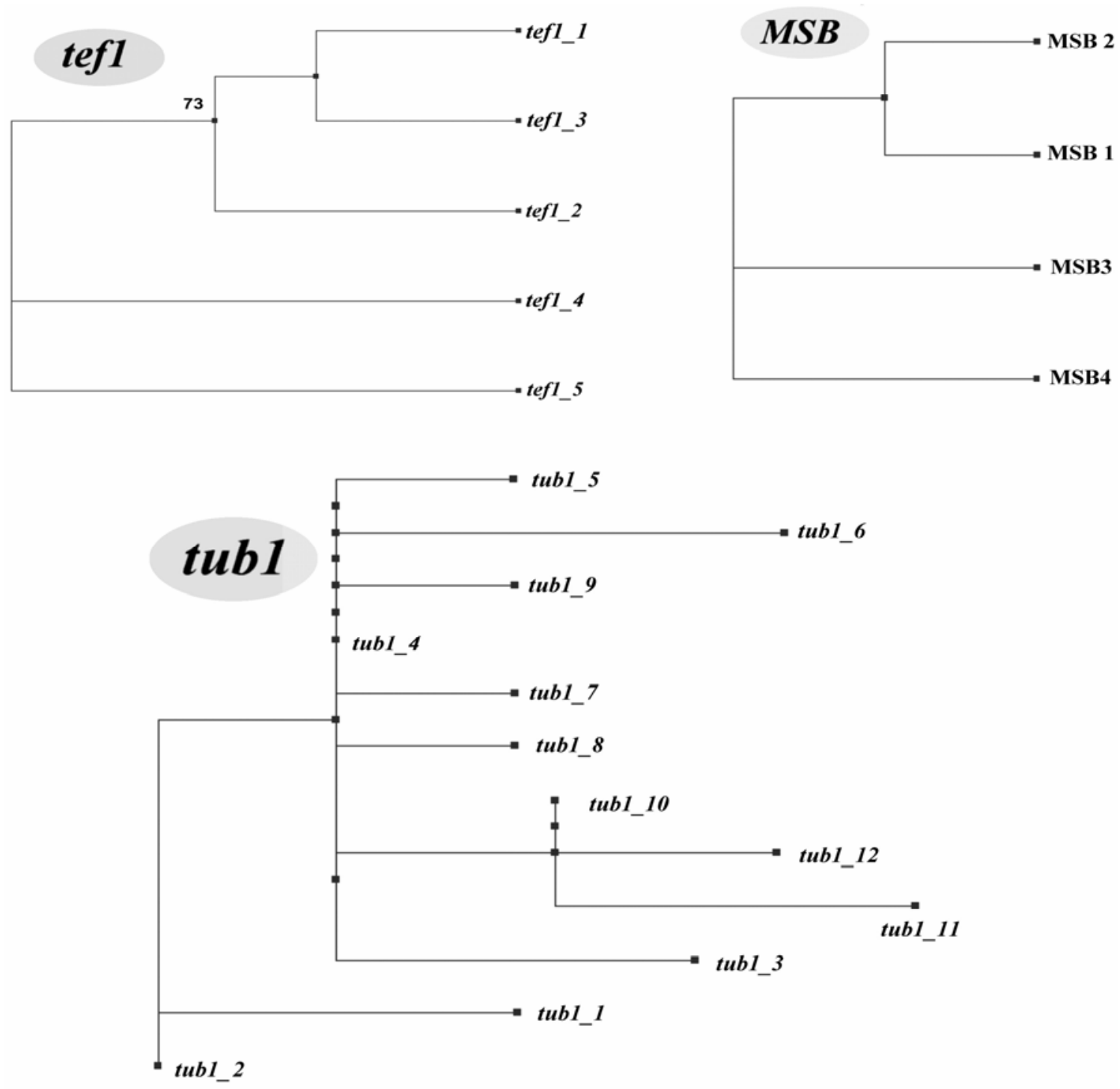

Fig. 2. Maximum likelihood analysis of gene phylogenies for $t e f 1$, tubl, and MSB1. Only one sequence per haplotype was used for the analysis. The HKI model was used for all three genes. Numbers over branches indicate bootstrap supports (not shown below 50). 
shown in Figure 3, indeed indicate that the sum of the minor haplotypes display a different ratio of the individual transposon genotypes than the population containing the major gene haplotype. However, since the two samples contain a significantly different number of strains, this finding is not statistically supported.

Recombination in the Hungarian $B$. cinerea population. The data above revealed incongruence between different gene trees, suggesting recombination within the Hungarian population of $B$. cinerea. Since the three genes used (MSB1, tefl, and tubl) did not contain sufficient genetic variation, we used microsatellites to test recombination. To this end, we used five microsatellites of $B$. cinerea $(\mathrm{Bc} 2, \mathrm{Bc} 3, \mathrm{Bc} 6, \mathrm{Bc} 7$, and $\mathrm{Bc} 10)$. They yielded 6, 6, 6, 11, and 13 fragments of different length, respectively. Their occurrence per strain resulted in 55 different microsatellite genotypes. When these genotypes were compared with the gene haplotypes (Fig. 3), no correlation with any of them was obtained (data not shown). Also, no correlation was obtained with either the geographic origin of the isolates or the year of isolation (data not shown).

To test for recombination, we used the $I_{A}$ test on a subset of clone-corrected data (i.e., individuals with identical genotypes at the five microsatellites were excluded so that each multilocus genotype was represented by only one individual [31]). The results from this test $\left(\mathrm{I}_{\mathrm{A}}=0.166 ; P<0.01\right)$ revealed that panmixia alone cannot be inferred from the data and that some clonality must be present. Similar values were obtained when only the isolates from 2003 or 2004 were subjected to the $\mathrm{I}_{\mathrm{A}}$ test $\left(\mathrm{I}_{\mathrm{A}}=0.145\right.$ and $0.187 ; P<0.01$ in both cases, respectively).

We also applied two tests on a concatenated set of our gene sequences, i.e., the maximum chi-square test and the phi test. The hypothesis of recombination was confirmed by both of them, i.e., the maximum chi-square test gave a value of $20.43(P=0.009)$; and the phi test resulted in $P=9.564 \times 10^{-4}$.

Thus, the application of three different criteria and two different sets of genetic markers consistently indicates that the population of Hungarian isolates of B. cinerea in the Eger wine region undergoes sexual reproduction.

Genetic drift within the Hungarian $B$. cinerea population. Because of the absence of complete panmixia, we computed the $\mathrm{F}_{\mathrm{ST}}$ values for all transposon populations and for all geographic populations. Qualitatively, an $\mathrm{F}_{\mathrm{ST}}$ value within the range of 0.05 to 0.15 indicates moderate differentiation (=fixation of characters), within 0.15 to 0.25 indicates great differentiation, and greater than 0.25 indicates very great differentiation (34). As shown in Table $3, \mathrm{~F}_{\mathrm{ST}}$ values between geographic populations were in the range of 0.018 to 0.077 , indicating no or only very low fixation. A similar analysis of the four transposing populations showed low differentiation between most of them. Only the examination of the $\mathrm{F}_{\mathrm{ST}}$ values between Flipper and vacuma - and less between transposa and vacuma - exhibited a moderate level of fixation ( 0.11 and 0.09 , respectively).

\section{DISCUSSION}

The results presented in this paper demonstrate that the Hungarian wine-pathogenic population of $B$. cinerea exhibits significant recombination. Whether or not recombination occurs in $B$. cinerea has been a matter of debate for many years, mainly because of the detection of only asexual propagation (=conidiation) on the plants. Yet, the use of rigorous genetic tests such as linkage disequilibrium (12) clearly showed that a $B$. cinerea population from France indeed propagated by sexual recombination. On the other hand, Ma and Michailides (22), studying Californian populations of $B$. cinerea, obtained essentially contrary data suggesting clonality. The difference between these studies could be due to the different geography of the study or the use of different genetic markers (gene restriction fragments versus random amplified polymorphic DNA analysis, respectively). In the present study, a
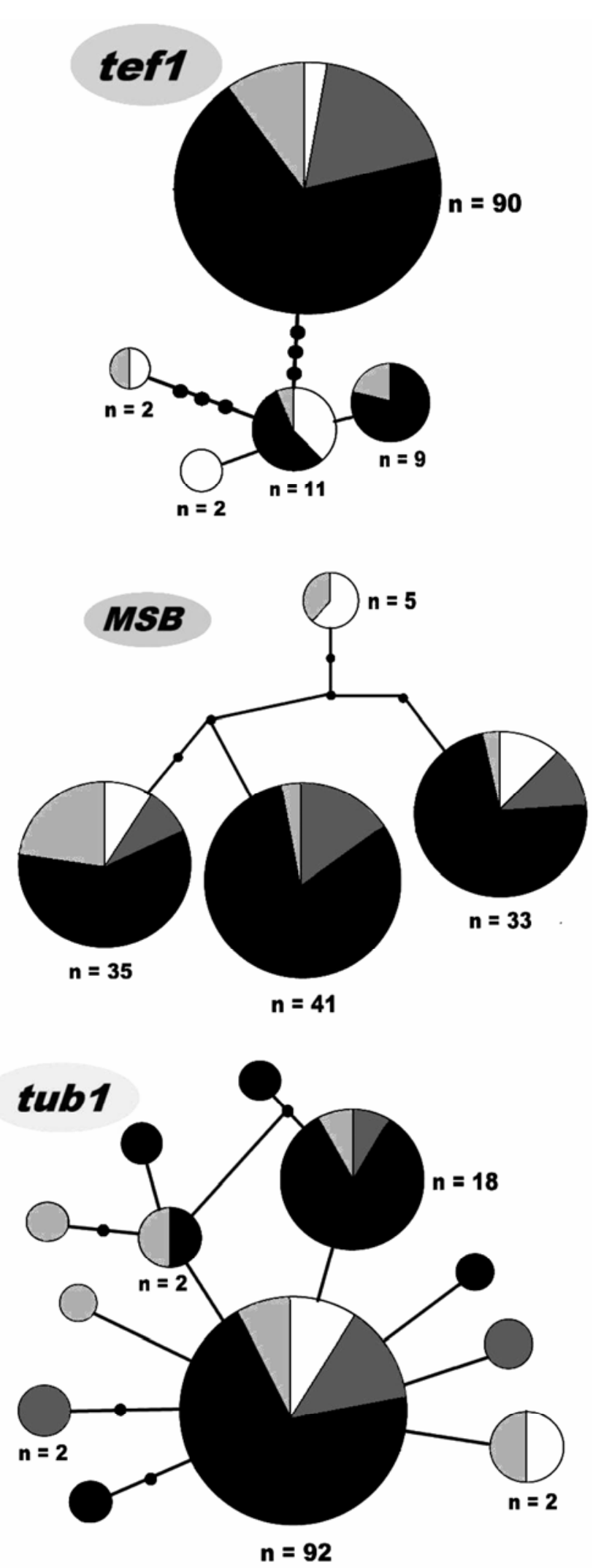

Fig. 3. Haplotype networks for $t e f 1, t u b 1$, and $M S B 1$, generated by statistical parsimony. $\mathrm{n}$ indicates number of isolates for each haplotypes, circles without $\mathrm{n}$ number consisted in single haplotypes only. Dots at the lines connecting haplotypes separate single mutations (= a line separated by no dot indicates a single mutation; one separated by a single dot indicates two mutations, etc.). Light gray sectors specify the proportion of isolates containing only Boty; and dark gray sectors specify isolates containing only Flipper. Black and white sectors indicate the proportion of isolates containing both and neither of them, respectively. 
very high degree of linkage disequilibrium was shown both by analyses of gene sequences as well as of microsatellites, which minimizes a potential bias. Fournier and Giraud (7) also provided evidence for recombination in a French population of $B$. cinerea. Therefore, although the geographic argument still cannot be refuted, it appears that the European populations of $B$. cinerea indeed perform recombination by sexual reproduction. However, the $I_{A}$ test also suggested an element of clonality. A corresponding analysis of the fixation index revealed that indeed the vacuma population exhibited some differentiation from both the Flipper and the transposa populations, which may explain these results. We could also detect some (albeit very moderate) differentiation between some geographic populations, particularly between II and IV and IV and V.

Previously, B. cinerea was believed to consist of two sympatric species, of which one (=transposa) contains both Flipper and Boty and the other (=vacuma) neither of them (12). vacuma and transposa were also found to significantly differ in phenotype such as conidial size, growth rates, and resistance against vinclozolin and diethofencarb (11). However, Fournier et al. (8), using GCPSR, provided the first evidence for two different sibling species of $B$. cinerea which they named group I and group II. In comparison to the vacuma and transposa concept, $B$. cinerea group I indeed contained only vacuma isolates. The results from the present study show that the $B$. cinerea populations in the Eger wine grape growing region in Hungary exhibit an unusual transposon composition; while the majority of isolates indeed were transposa, vacuma isolates were present in about the same percentage as isolates containing only Boty or only Flipper. The presence of only Flipper in B. cinerea has so far not been detected in isolates from California (22) and Chile (27), and seems to be strongly underrepresented in samples from England, France, and Tunisia $(1,3)$. In contrast, a more recent study on $B$. cinerea isolates from Croatia (26) reported $26 \%$ of the isolates to contain only Flipper, whereas $41 \%$ were transposa. The higher number of isolates containing only Flipper raises the question of how these strains evolved, i.e., from transposa strains by loss of Boty, or from vacuma strains by gain of Flipper. Munoz et al. (27) attempted to explain the occurrence of Boty isolates in a Chilean population by crosses between vacuma and transposa, or by the existence of an invading population of the vacuma group. Because of the high linkage equilibrium in our genetic markers, we cannot distinguish between these two possibilities. In theory, the roughly equal number of Boty, Flipper, and vacuma isolates would be compatible with the speculation of a recombination of transposa with an invading population of vacuma. However, our data showed that the vacuma population exhibits the highest level of clonality of all transposon populations which makes this explanation rather unlikely. In any case, the evidence from our data, i.e., that $B$. cinerea group II also contains isolates which only contain Flipper or Boty, further underlines that the presence or absence of either transposon is not a valid character for speciation in B. cinerea.

In summary, our data underline a significant genetic variability within the Hungarian population of $B$. cinerea. From a phytopathological perspective, a more detailed insight into the mechanisms leading to the formation of the "Flipper-only" isolates will be worthwhile, as neither its physiological characters (fungicide

TABLE 3. $\mathrm{F}_{\mathrm{ST}}$ values for microsatellite markers between different geographic populations in the Eger wine region, Hungary

\begin{tabular}{lcccc}
\hline Location & II & III & IV & V \\
\hline I & 0.018 & 0.029 & 0.025 & 0.039 \\
II & & 0.038 & 0.058 & 0.043 \\
III & & & 0.038 & 0.024 \\
IV & & & & 0.077 \\
\hline
\end{tabular}

resistance and appearance during pathogenesis) nor the contribution of these isolates to the population structure of the pathogen have been examined so far.

\section{ACKNOWLEDGMENTS}

This work was supported by grants from the Ministry of Agriculture and Rural Development (33013/2003 and 46024/2004) and the National Office for Research and Technology (NKTH; A2-2006-0017). L. Karaffa was supported by the OTKA (Hungarian Scientific Research Fund; F042602 and K67667) and a Bolyai János Research Scholarship (BO/00107/06). E. Fekete and E. Sándor are recipients of an OTKA postdoctoral scholarship (D048617) and a Bolyai János Research Scholarship (BO/00446/04), respectively.

\section{LITERATURE CITED}

1. Albertini, C., Thebaud, G., Fournier, E., and Leroux, P. 2002. Eburicol $14 \alpha$-demethylase gene (CYP51) polymorphism and speciation in Botrytis cinerea. Mycol. Res. 106:1171-1178.

2. Beever, R. E., and Weeds, P. 2004. Pages: 29-52 in: Botrytis: Biology, Pathology and Control. Y. Elad, B. Williamson, and P. Tudzynski, eds. Kluwer Academic Publishers, Dordrecht, the Netherlands.

3. Ben Ahmed, D., and Hamada, W. 2005. Genetic diversity of some Tunisian Botrytis cinerea isolates using molecular markers. Phytopathol. Mediterr. 44:300-306.

4. Clement, M., Posada, D., and Crandall, K. 2000. TCS: A computer program to estimate gene genealogies. Mol. Ecol. 9:1657-1660.

5. Diolez, A., Marches, F., Fortini, D., and Brygoo, Y. 1995. Boty, a longterminal-repeat retroelement in the phytopathogenic fungus Botrytis cinerea. Appl. Environ. Microbiol. 61:103-108.

6. Farethra, F., and Pollastro, S. 1996. Genetic studies of the phytopathogenic fungus Botryotinia fuckeliana (Botrytis cinerea) by analysis of ordered tetrads. Mycol. Res. 100:620-624.

7. Fournier, E., and Giraud, T. 2008. Sympatric genetic differentiation of a generalist pathogenic fungus, Botrytis cinerea, on two different host plants, grapevine and bramble. J. Evol. Biol. 21:122-132.

8. Fournier, E., Giraud, T., Albertini, C., and Brygoo, Y. 2005. Partition of the Botrytis cinerea complex in France using multiple gene genealogies. Mycologia 97:1251-1267.

9. Fournier, E., Giraud, T., Loiesau, A., Vautrin, D., Estoup, A., Solignac, M., Cornuet, J. M., and Brygoo, Y. 2002. Characterization of nine polymorphic microsatellite loci in the fungus Botrytis cinerea (Ascomycota). Mol. Ecol. Notes 2:253-255.

10. Giraud, T., Fortini, D., Levis, C., and Brygoo, Y. 1998. The minisatellite MSB1 in the fungus Botrytis cinerea, probably mutates by slippage. Mol. Biol. Evol. 15:1524-1531.

11. Giraud, T., Fortini, D., Levis, C., Lamarque, C., Leroux, P., LoBuqlio, K., and Brygoo, Y. 1999. Two siblings species of the Botrytis cinerea complex, transposa and vacuma, are found in sympatry on numerous host plants. Phytopathology 89:967-973.

12. Giraud, T., Fortini, D., Levis, C., Leroux, P., and Brygoo, Y. 1997. RFLP markers show genetic recombination in Botryotinia fuckeliana (Botrytis cinerea) and transposable elements reveal two sympatric species. Mol. Biol. Evol. 14:1177-1185.

13. Guindon, S., and Gascuel, O. 2003. PhyML-A simple, fast, and accurate algorithm to estimate large phylogenies by maximum likelihood. System. Biol. 52:696-704.

14. Huelsenbeck, J. P., and Ronquist, F. 2001. MRBAYES: Bayesian inference of phylogenetic trees. Bioinformatics 17:754-755.

15. Huson, D. H., and Bryant, D. 2006. Application of phylogenetic networks in evolutionary studies. Mol. Biol. Evol. 23:254-267.

16. Jaklitsch, W. M., Komon, M., Kubicek, C. P., and Druzhinina, I. S. 2006. Hypocrea crystalligena sp. nov., a common European species with a white-spored Trichoderma anamorph. Mycologia 98:499-513.

17. Jarvis, W. R. 1980. Taxonomy. Pages 1-18 in: The Biology of Botrytis. J. R. Coley-Smith, K. Verhoeff, and W. R. Jarvis, eds. Academic Press, London.

18. Kasuga, T., White, T. J., Koenig, G., McEwen, J., Restrepo, A., Castañeda, E., Da Silva Lacaz, C., Heins-Vaccari, E. M., De Freitas, R. S., Zancopé-Oliveira, R. M., Qin, Z., Negroni, R., Carter, D. A., Mikami, Y., Tamura, M., Taylor, M. L., Miller, G. F., Poonwan, N., and Taylor, J. W. 2003. Phylogeography of the fungal pathogen Histoplasma capsulatum. Mol. Ecol. 12:3383-3401.

19. Kumar, S., Tamura, K., and Nei, M. 2003. MEGA3: Integrated software for molecular evolutionary genetics analysis and sequence alignment. Brief Bioinform. 5:150-63.

20. Levis, C., Fortini, D., and Brygoo, Y. 1997. Flipper, a mobile Fot1-like 
transposable element in Botrytis cinerea. Mol. Gen. Genet. 254:674-680.

21. Lorbeer, J. W. 1980.Variation in Botrytis and Botryotinia. Pages 19-39 in: The Biology of Botrytis. J. R. Coley-Smith, K. Verhoeff, and W. R. Jarvis, eds. Academic Press, London.

22. Ma, Z., and Michailides, T. J. 2007. Approaches for eliminating PCR inhibitors and designing PCR primers for the detection of phytopathogenic fungi. Crop Prot. 26:145-161.

23. Martinez, F., Blancard, D., Lecomte, P., Levis, C., Dubos, B., and Fermaud, M. 2003. Phenotypic differences between vacuma and transposa subpopulations of Botrytis cinerea. Eur. J. Plant Pathol. 109:479-488.

24. Martinez, F., Dubos, B., and Fermaud, M., 2005. The role of saprotrophy and virulence in the population dynamics of Botrytis cinerea in vine yards. Phytopathology 95:692-700.

25. Maynard Smith, J. 1992. Analyzing the mosaic structure of genes. J. Mol. Evol. 35:126-129.

26. Milicevic, T., Topolovec-Pintaric, S., Cvjetkovic, B., Ivic, D., and Duralija, B. 2006. Sympatric populations of Botrytis cinerea on strawberries based on the content of transposable elements and their connection with resistance to botryticides. Acta Horticult. 708:115-118.

27. Munoz, G., Hinrichsen, P., Brygoo, Y., and Giraud, T. 2002. Genetic characterisation of Botrytis cinerea populations in Chile. Mycol. Res. 106:594-601.
28. Nei, M. 1973. Analysis of gene diversity in subdivided populations. Proc. Natl. Acad. Sci. USA 70:3321-3323.

29. Nicholas, K. B., and Nicholas, H. B., Jr. 1997. Genedoc: A tool for editing and annotating multiple sequence alignments. Published online by the National Resource for Biomedical Supercomputing (NRBSC), Pittsburgh.

30. Piganeau, G., Gardner, M., and Eyre-Walker, A. 2004. A broad survey of recombination in animal mitochondria. Mol. Biol. Evol. 21:23192325.

31. Pringle, A., Baker, D. M., Platt, J. L., Wares, J. P., Latge, J. P., and Taylor, J. W. 2005. Cryptic speciation in the cosmopolitan and clonal human pathogenic fungus Aspergillus fumigatus. Evolution 59:1886-1899.

32. Rozas, J., Sanchez-Delbarrio, J. C., Messeguer, X., and Rozas, R. 2003. DnaSP, DNA polymorphism analyses by the coalescent and other methods. Bioinformatics 19:2496-2497.

33. Thompson, J. D., Gibson, T. J., Plewniak, F., Jeanmougin, F., and Higgins, D. G. 1997. The ClustalX windows interface: Flexible strategies for multiple sequence alignment aided by quality analysis tools. Nucleic Acids Res. 25:4876-4882.

34. Wright, S. 1978. Variability Within and Among Natural Populations. Evolution and Genetics of Populations, Vol. 4. University of Chicago Press, Chicago, IL. 